\section{COMPARISON BETWEEN ACTUAL DAILY AND RECOMMENDED NUTRIENT INTAKES, DURING THE FIRST TWO MONTHS OF LIFE, IN PRETERM ELBW INFANTS}

L. Corvaglia, P. Montesano, M. De Giorgi, A. Aceti, E. Mariani, G. Faldella

Neonatology and Neonatal Intensive Care Unit S. Orsola-Malpighi Hospital - University of Bologna, Bologna, Italy

Background and Aims: Recently, the European Society of Pediatric Gastroenterology Hepatology and Nutrition has reviewed the guidelines for preterm infants' enteral nutrition, recommending a 4.8-6.6 g/ $/ \mathrm{kg} /$ day lipid intake, and a $4-4.5 \mathrm{~g} / \mathrm{kg} /$ day protein intake for infants weighing less than $1 \mathrm{~kg}$ and $3.5-4 \mathrm{~g} / \mathrm{kg} /$ day for infants weighing $1-1.800 \mathrm{~kg}$.

However, nutritional goals are difficult to achieve in this population, with the risk of subsequent short and long-term adverse effects.

Our aim was to compare daily the actual and recommended protein and fat intakes during the first two months of life in ELBW preterm infants.

Methods: Actual protein and fat parenteral and enteral intakes ( $g /$ day) were evaluated daily in 8 ELBW infants. Human milk (HM) content was analyzed by Near-Infrared-Reflectance-Analysis; protein and fat provided by HM fortifier and formula were recorded. Total daily protein and fat intake was then calculated.

Results: Actual protein and fat intakes were lower than recommended along all the first two months of life. Specifically, a median 30\% deficit (range 22$38 \%$ ) in protein intake and $50 \%$ deficit (range 31 $63 \%$ ) in fat intake was documented.

Conclusions: Preliminary results of our study show that, in preterm infants, it is difficult to achieve nutritional goals, due to inter-individual variability in clinical status and to the different composition of $\mathrm{HM}$. This inadequate nutrient intake determines a cumulate delay in the extrauterine growth, which represents one of the most important problem for preterm infants.

\section{VITAMIN A MODIFIES THE EXPRESSION OF ANTIOXIDANT ENZYMES IN THE DEVELOPING LUNG}

\author{
R. Carvalho', M.L. Pinto ${ }^{2}$, A. Coelho', \\ C. Gonçalves ${ }^{3}$, V. Bairos ${ }^{3}$ \\ ${ }^{1}$ Veterinary Sciences, ${ }^{2}$ University of Trás-os- \\ Montes e Alto Douro, Vila Real, ${ }^{3}$ Histology \\ and Embriology, Faculdade de Medicina da \\ Universidade de Coimbra, Coimbra, Portugal
}

Retinol has proven to be an essential molecule during lung fetal development, but its effects on the pulmonary antioxidant system are still unknown. At birth, there is a major increase of reactive oxygen species due to the exposure to oxygen, which may lead to cellular damage. This increase is particularly important in the premature, due to the antioxidant system immaturity. To investigate the retinol influence on lung's antioxidant enzymes we conducted an in vivo study. Pregnant mice were subjected to subcutaneous administration of vitamin A on the $12^{\text {th }}$ gestational day. Lungs from control and treated animals were collected daily from the $15^{\text {th }}$ gestational day till the $3^{\text {th }}$ day of life. Catalase was measured by the polarographic method and glutathione peroxidase and superoxide dismutase by spectrometry. Ours results show a positive effect of vitamin A administration on both catalase and glutathione peroxidase enzymes. A statistical significance of catalase expression was reached at $16^{\text {th }}$ gestational day in vitamin A-exposed lungs. An identical difference in glutathione peroxidase expression was registered at the $15^{\text {th }}$ and $16^{\text {th }}$ gestational days. We have previously demonstrated that catalase and glutathione peroxidase levels are higher in the lungs of males in the same timeperiod, a feature that was not altered by vitamin A administration. Previous studies from our group also showed that maternal administration of vitamin A results in an enhancement of lung organogenesis, which can be achieved, in part, by stimulating the lung's antioxidant system, as occurs with the surfactant system, without eliminating free radicals physiological functions. 